\title{
Probing the thermal effects of voltage hysteresis in anionic-redox- based lithium-rich cathodes using isothermal calorimetry
}

\author{
Gaurav Assat, ${ }^{1,2,3}$ Stephen L. Glazier, ${ }^{4}$ Charles Delacourt, ${ }^{*, 2,5}$ Jean-Marie Tarascon*,1,2,3 \\ ${ }^{1}$ Collège de France, Chimie du Solide et de l'Energie - UMR 8260 CNRS, 11 Place Marcelin Berthelot, 75005 \\ Paris, France \\ ${ }^{2}$ Réseau sur le Stockage Electrochimique de l'Energie (RS2E) - FR CNRS 3459, 80039 Amiens Cedex, France \\ ${ }^{3}$ Sorbonne Université - UPMC Paris 06, 4 Place Jussieu, 75005 Paris, France \\ ${ }^{4}$ Department of Physics and Atmospheric Science, Dalhousie University, Halifax, N. S., B3H 4R2, Canada \\ ${ }^{5}$ Laboratoire de Réactivité et Chimie des Solides (LRCS) - UMR CNRS 7314, Université de Picardie Jules \\ Verne, 33 rue Saint Leu, 80039 Amiens Cedex, France

\footnotetext{
* Corresponding authors:

C. Delacourt: charles.delacourt@u-picardie.fr

J.-M. Tarascon: jean-marie.tarascon@college-de-france.fr
} 


\begin{abstract}
The commercialization of high-energy batteries with Li-rich cathode materials exhibiting combined cationic/anionic redox processes awaits the elimination of certain practical bottlenecks. Among these, large voltage hysteresis remains the most obscure from a fundamental thermochemical perspective. Here we study this issue by directly measuring via isothermal calorimetry the heat generated by $\mathrm{Li} / \mathrm{Li}_{2} \mathrm{Ru}_{0.75} \mathrm{Sn}_{0.25} \mathrm{O}_{3}$ (Li/LRSO) cells during various cycling conditions, with LRSO being a 'model' Li-rich layered cathode. We show how this heat thermodynamically relates to the lost electrical work that is crucial for practical applications. We further reveal that anionic redox on charging and discharging adopts different metastable paths having non-identical enthalpy potentials such that the overall Li content no longer remains the unique reaction coordinate, unlike in fully path-reversible cationic redox. We elucidate how quasistatic voltage hysteresis is related with heat dissipated due to non-equilibrium entropy production. Overall, this study establishes the great benefits of isothermal calorimetry for enabling energy efficient electrode materials in next-generation batteries.
\end{abstract}




\section{Introduction}

Increasing the energy density of lithium-ion batteries requires denser positive electrodes with higher voltage and/or capacity. $\mathrm{Li}_{2} \mathrm{MnO}_{3}$-based Li-rich layered oxides (Li-rich NMCs), such as $\mathrm{Li}_{1.2} \mathrm{Ni}_{0.13} \mathrm{Mn}_{0.54} \mathrm{Co}_{0.13} \mathrm{O}_{2}$, can deliver specific capacities above $270 \mathrm{mAh} \mathrm{g}$ to reach $1000 \mathrm{Wh} \mathrm{kg}^{-1}$ of specific energy at the material level. Despite their slightly lower crystalline density than today's Li-stoichiometric Ni-based layered oxides (NMCs and NCA), Li-rich cathodes remain very promising for the long-term, as we will need to move away from Ni-rich towards Mn-rich materials (Mn is inexpensive and environmentally benign) without compromising the energy density. ${ }^{1-3}$ The high capacity of Li-rich NMCs stems from cumulative anionic and cationic bulk redox processes. ${ }^{4-}$ ${ }^{6}$. However, these electrodes currently fall short in other performance metrics ${ }^{7}$ because of large voltage hysteresis $^{5,8}$, sluggish kinetics ${ }^{5,9,10}$, and gradual voltage fade ${ }^{11}$. These issues are concomitant with anionic redox activity $^{7}-$ the very same feature that enhances capacity. Therefore, further investigations are needed to fundamentally understand the overall anionic redox process, which constitutes not just of electron removal from oxygen-based electronic states, but also the ensuing (local) structural/bonding rearrangements. ${ }^{7}$

The undesirable issue of voltage hysteresis in rechargeable batteries leads to energy inefficiency, presumably dissipated as heat, consequently imposing an additional energy cost on the end-users. ${ }^{12}$ Voltage hysteresis would also complicate the state of charge (SoC) and thermal management of such batteries. Li-rich NMCs show a relatively large difference between charge/discharge voltages ( 400-500 mV after first cycle, $\sim 87 \%$ energy efficiency, Figure 1$)$. This gap persists over cycling, even at extremely low rates $(C / 300)^{10}$, at high temperatures $(55$ or 85 $\left.{ }^{\circ} \mathrm{C}\right)^{13}$, and after long relaxation periods (100 hours) ${ }^{10}$. Such behaviour therefore cannot be explained by simple electrochemical kinetics that fails to explain the observed path dependence and quasistatic hysteresis. Interestingly and similar to Li-rich NMCs, many other newly-discovered Li- and Na-based materials with reversible anionic redox also suffer from voltage hysteresis. ${ }^{7}$ These include 
layered $\mathrm{Li}_{2} \mathrm{Ru}_{1-x} \mathrm{Sn}_{x} \mathrm{O}_{3}{ }^{9}$ (Figure 1), $\mathrm{Na}_{2 / 3}\left[\mathrm{Mn}_{1-\chi} \mathrm{Mg}_{x}\right] \mathrm{O}_{2}{ }^{14}$, and $\mathrm{Na}_{2} \mathrm{RuO}_{3}{ }^{15}$ as well as disordered $\mathrm{Li}_{1.2} \mathrm{Mn}_{0.4} \mathrm{Ti}_{0.4} \mathrm{O}_{2}{ }^{16}$ and $\mathrm{Li}_{2} \mathrm{MnO}_{2} \mathrm{~F}^{17}$.

Only a few studies have attempted to understand the origin of voltage hysteresis in this class of cathodes. These include electrochemical measurements in different voltage windows to identify correlated differential capacity $(d Q / d V)$ peaks ${ }^{5,8,11,18,19},{ }^{6} \mathrm{Li}$ nuclear magnetic resonance to observe path-dependence in lithium site occupation ${ }^{20}$, X-ray diffraction to claim back-and-forth (partially reversible) transition-metal migration ${ }^{21}$, and bulk X-ray spectroscopies ${ }^{5,22,23}$ to demonstrate the absence of hysteresis in the potentials at which transition-metals show redox-activity, unlike for the hysteretic oxygen redox process. A couple of phenomenological models assuming either a Li-driven phase change ${ }^{10}$ or an asymmetry in transition-metal migration ${ }^{24}$ were also conceived. Despite such widespread efforts, the general thermodynamic mechanism behind voltage hysteresis and its thermal effects remain unclear. Two questions need answering: (i) how exactly is the lost energy dissipated as heat so that it can be better managed/predicted? and (ii) what is the underlying mechanism along with the corresponding thermochemical conditions that lead to hysteresis?

In light of this, we adopt a different approach herein and perform isothermal calorimetry measurements during the cycling of a 'model' Li-rich layered cathode $-\mathrm{Li}_{2} \mathrm{Ru}_{0.75} \mathrm{Sn}_{0.25} \mathrm{O}_{3}$ (LRSO) or $\mathrm{Li}\left[\mathrm{Li}_{0.33} \mathrm{Ru}_{0.5} \mathrm{Sn}_{0.17}\right] \mathrm{O}_{2}$. This high capacity $\left(\sim 250 \mathrm{mAh} \mathrm{g}{ }^{-1}\right.$ reversibly) material, although only suitable for niche applications without cost batteries (e.g. space), shows remarkable structural and electrochemical similarities with the practically important Li-rich NMCs (Figure 1). ${ }^{9,25}$. Overall, it serves as a simplified 'model' compound to understand the general properties of Li-rich layered electrodes. The magnitude of voltage hysteresis in LRSO $(\sim 200-300 \mathrm{mV})$ is lower than Li-rich NMCs but the role of anionic redox activity is particularly similar, such that high potential charging triggers the onset of hysteresis. ${ }^{9,23}$ Using isothermal calorimetry in this work, we report the heat generation during different electrochemical stages of LRSO, starting with the classical cationic redox step in first charge followed by the voltage plateau accompanying oxygen redox and finally 
[Figure 1]

Figure 1 | Comparison of voltage profiles of Li-rich NMC and LRSO. The first cycle (a and b) displays a two-stepped charge followed by a sloped discharge. The labels "C" and " $A$ " denote respectively the cationic and anionic redox processes. The later cycles (c and $\mathbf{d}$ ) display a quasistatic voltage hysteresis, leading to an energy inefficiency that is equivalent to the shaded area between the charge and discharge profiles. In $\mathbf{a}$ and $\mathbf{b}$, the materials do not get back to their initial states after the first cycle, unlike for the already-formed materials in $\mathbf{c}$ and $\mathbf{d}$.

\section{Thermodynamic Considerations \\ Thermodynamic Considerations}

the hysteretic sloped voltage profiles that stabilize over cycling. These measurements allow us to discuss the thermodynamics in terms of enthalpy change, entropy change, and entropy production. We highlight the thermal asymmetry in the charge vs. discharge paths as well as the difficulties in locating the true equilibrium properties of such hysteretic materials. A multi-step reaction scheme for the solid-state oxygen redox reaction is finally proposed to comprehend the results.

The heat generated by an electrochemical cell in isothermal and isobaric conditions is described in the Methods section. ${ }^{26,27}$ Because of large voltage hysteresis and path-dependence in Li-rich cathodes ${ }^{5,9}$, the position of their equilibrium potential $U$ cannot simply be assumed in the middle of the charge and discharge, unlike in classical path-reversible intercalation materials. We therefore need a different approach herein. Neglecting heat of mixing ${ }^{27,28}$ and correcting for minor parasitic heat flow (see details in Methods), the energy balance can be recast as

$$
V-\frac{\left.\dot{q}\right|_{T, P}}{I}=U-T \frac{\partial U}{\partial T}=U_{H}
$$


with

$$
U_{H}=-\frac{\Delta H}{n F}
$$

where $V$ is the cell voltage, $\left.\dot{q}\right|_{T, P}$ is the heat generation rate (positive if heat is released by cell), $I$ is the current (positive for charging), $T$ is the temperature, $P$ is the surrounding pressure, $n$ is the number of electrons involved in electrode reaction, $F$ is the Faraday's constant, and $U_{H}$ is the so-called "enthalpy potential". ${ }^{27}$ Just as $U$ is directly related to the partial change in Gibbs free energy of the cell as its SoC is varied $\left(U=-\frac{\Delta G}{n F}\right), U_{H}$ is directly related to the partial change in the enthalpy $H$ of the cell. In other words, $U_{H}$ captures the energy change while $U$ includes the entropy change as well. $U_{H}$ can thus simply be obtained by measuring $V, I$, and $\left.\dot{q}\right|_{T, P}$. Knowing $U_{H}$ can help in predicting the heat generation under operating conditions for proper thermal management of batteries.

Integrating

Equation [1] over a closed path, such as a charge-discharge cycle after which the material returns to its original state, gives

$$
\oint_{\text {cycle }} I V d t=\left.\oint_{\text {cycle }} \dot{q}\right|_{T, P} d t
$$

because enthalpy $H$ is a state variable and cancels out over a stable cycle as follows

$$
\oint_{\text {cycle }} d H=-n F \oint_{\text {cycle }} U_{H} d x=0
$$


Equation [3] demonstrates that the net electrical work due to hysteretic voltage profiles is dissipated as waste heat. Note that the $U_{H}$ measured via

Equation [1] (under applied current) can be path dependent, i.e. different on charge vs. discharge for the same $\mathrm{SoC}$, if the materials inside the cell remain as metastable phases under dynamic conditions away from the true equilibrium. Nevertheless, even in that non-equilibrium case, $U_{H}$ over a stable cycle (i.e. having no net change in $H$ ) is constrained by Equation [4] and therefore cannot form a hysteretic loop versus Li composition $x$, as schematized in Supp. Figure 1. Note that Li-rich materials do not have a perfectly stable cycle because of gradual voltage fade. However, these changes will turn out to be negligible for LRSO later in the paper.

In addition to the above energy balance, the Second Law of Thermodynamics relates the entropy change to the heat exchange as ${ }^{29}$

$$
\begin{gathered}
\frac{\left.\dot{q}\right|_{T, P}}{T}=-\dot{S}+\dot{\sigma}_{\text {prod }} \\
\dot{\sigma}_{\text {prod }} \geq 0
\end{gathered}
$$

where $\dot{S}$ is the rate of entropy change of the cell and $\dot{\sigma}_{\text {prod }}$ is the rate of entropy production due to the irreversible processes inside the cell. $\dot{\sigma}_{\text {prod }}$ is close to zero only over a thermodynamically reversible path. Since entropy is a state variable, its integration over a closed path (e.g. a stable charge-discharge cycle) becomes

$$
\oint_{\text {cycle }} \dot{S} d t=\oint_{\text {cycle }} \frac{d S}{d x} d x=0
$$


Consequently, the integration of Equation [5] over a closed path, when combined with Equation [3], gives

$$
\oint_{\text {cycle }} I V d t=\left.\oint_{\text {cycle }} \dot{q}\right|_{T, P} d t=\oint_{\text {cycle }} T \dot{\sigma}_{\text {prod }} d t \geq 0
$$

Equation [7]

The overall voltage hysteresis (both resistive and current-independent in nature) is therefore a measure of entropy production that is dissipated as heat. Entropy production in the realm of chemical reactions $^{29}$ is further described along-with Supp. Figure 2.

\section{Isothermal calorimetry results for the $3.5 \mathrm{~V}$ cationic redox}

Initial electrochemical de-intercalation of up to $0.75 \mathrm{Li}$ per formula unit from $\mathrm{Li}_{2} \mathrm{Ru}_{0.75} \mathrm{Sn}_{0.25} \mathrm{O}_{3}$ (LRSO) takes place around $3.5 \mathrm{~V}$ with the oxidation of primarily $\mathrm{Ru}$-based electronic states (hence termed cationic redox). This redox step is completely reversible. ${ }^{9,23}$ Isothermal calorimetry measurements at $40{ }^{\circ} \mathrm{C}$ (see Methods for details) were performed over this step in coin-type Li half-cells at different $\mathrm{C}$-rates, ranging from $\mathrm{C} / 20$ to $\mathrm{C} / 5$. Figure 2a shows the resulting voltage profiles, which were used along with the simultaneously measured heat flow rates to obtain the profiles of enthalpy potential $U_{H}$ using

Equation [1], as shown in Figure 2b for the slowest rate of $\mathrm{C} / 20$. The symmetric shape of the heat flow profiles (orange curves) arises because of reversible entropic heat, whereas the net positive shift is due to irreversible heat from overpotential (see Methods for further explanations). The calculated $U_{H}$ profiles (pink curves) superimpose neatly on charge and discharge, indicating that the enthalpy change is path independent (Supp. Figure 1a) and is only a function of the Li 
stoichiometry (SoC). Such behaviour is typical for classical electrochemical intercalation reactions. ${ }^{26,30}$ Understanding the exact shape of entropic heat flow is beyond the scope of this study, as it may be related to many aspects of the cathode material, such as the changes in $\mathrm{Ru}-\mathrm{Ru}$ bond distance ordering ${ }^{23}$, charge ordering, Li vacancy ordering, or the shape of the $d Q / d V$ profile (Supp. Figure 3).

The deviation (early drop, marked by green arrows) of the discharge $U_{H}$ profile from that on charge in Figure $2 \mathrm{~b}$ is likely because of concentration gradients and can be understood better by studying the effect of varying C-rates. The $U_{H}$ profiles obtained at different rates are compared in Figure 2c (see Supp. Figure 4 for the raw heat flow profiles). They converge over a broad range of SoCs for both charge and discharge, however with some rate-dependent discrepancies at the extremities, as highlighted with green backgrounds in Figure 2c. These kinetic effects arise from the formation and relaxation of concentration gradients, most likely in the solid phase due to limited lithium diffusion since the electrode is designed with a high ( $\sim 60 \%)$ porosity (electrode details in Methods), and the associated heat of mixing effects ${ }^{27,28}$ that were neglected in

Equation [1]. Nevertheless, the $U_{H}$ profiles at the slowest rate superimpose adequately and reflect the enthalpy change of the electrochemical reaction. Moreover, the voltage and heat profiles remain perfectly identical over consecutive cycles in this cationic redox regime (Supp. Figure 5).

Lastly, Figure 2d compares the net electrical work and the net heat generated from the cell over a complete cycle at different rates. The heat and work are positive and match with each other, in agreement with Equation [7]. Moreover, they exhibit a linear relationship with current that approaches close to zero as the current diminishes. Overall, the calorimetric analysis of the $3.5 \mathrm{~V}$ cationic redox step demonstrates its classical nature, i.e. it is path independent with minor quasistatic voltage hysteresis, and can be thermodynamically treated as a single-step electrochemical reaction. 
[Figure 2]

Figure 2 | Isothermal calorimetry characterization of the $3.5 \mathrm{~V}$ cationic redox step. Voltage profiles (a) obtained with LRSO cycled inside the calorimeter at $40{ }^{\circ} \mathrm{C}$ using CCCV protocols after a few formation cycles. The polarization gradually increases with increasing C-rates. For C/20 (b), the measured profiles of cell voltage (blue) and heat flow rate (orange) that were used to obtain the $\boldsymbol{U}_{\boldsymbol{H}}$ profiles (pink). $\boldsymbol{U}_{\boldsymbol{H}}$ lies above the equilibrium potential $\boldsymbol{U}$ located somewhere between the charge and discharge voltages. Therefore, the entropic term is such that the cell's entropy increases on charge (causing heat absorption) and decreases on discharge (causing heat release). (c) Convergence of $\boldsymbol{U}_{\boldsymbol{H}}$ profiles obtained at different rates, with green backgrounds highlighting the deviations possibly due to heat of mixing. As the C-rate increases, the charge $\boldsymbol{U}_{\boldsymbol{H}}$ profiles develop a small distortion at the onset of charge and show an early rise towards the end of charge. Similarly, the discharge $\boldsymbol{U}_{\boldsymbol{H}}$ profiles show an early drop towards the end of discharge. (d) Comparison of lost electrical work with dissipated waste heat over complete charge-discharge cycles. The slope and intercept are respectively related to the "SoC averaged" values of $\boldsymbol{R}_{\text {int }}$ and $\boldsymbol{V}_{\text {hyst }}$ in Equation [10]Error! Reference source not found.

\section{Isothermal calorimetry results for the $4.2 \mathrm{~V}$ anionic redox}

Further extraction of Li from LRSO takes place at the $4.2 \mathrm{~V}$ plateau (Figure 3a), which is charge-compensated by the oxidation of bulk lattice oxygen (electron removal from oxygen nonbonding levels, hence termed anionic redox). ${ }^{7,23}$ This step releases significantly larger heat than the preceding cationic redox (Figure $3 b$ ). Such a large heat release cannot simply be explained by electrochemical resistance, which we previously showed to remain small. ${ }^{9}$ We had additionally reported very slow equilibration dynamics, such that the open-circuit voltage (OCV) slowly relaxes away from the voltage under load for long periods of time. Note in this case that the direct determination of the entropic term $\frac{\partial U}{\partial T}$ by temperature-dependent potential measurements, as attempted for Li-rich $\mathrm{NMC}^{31}$, is questionable. Structure-wise, the anionic redox plateau leads to a loss of crystallinity along with the onset of local distortion, in contrast to the preceding cationic step. $^{23,25}$ Moreover, the subsequent discharge adopts a different sloped S-shaped path (Figure 3c). All these observations indicate that the $4.2 \mathrm{~V}$ plateau is an electrochemically-driven irreversible 
phase transformation (hereafter termed as 'activation') from the crystalline partially-charged phase to the disordered/distorted fully-charged one. This is reminiscent of conversion-type electrode materials and cannot be treated as a single-step electrochemical reaction. We therefore propose a two-step electrochemical/chemical (EC) mechanism to explain the anomalous heat generation. The electrochemical (E) step consists of electron removal from states derived primarily from oxygen (anionic oxidation) and the follow-up chemical (C) step is a stabilizing structural rearrangement, e.g. the distortion of $\mathrm{RuO}_{6}$ octahedra leading to the shortening of $\mathrm{O}-\mathrm{O}$ distances and/or metal-ion migration. $^{7,23,25}$ This stabilizing chemical step explains the large heat generation observed over the plateau.

The $U_{H}$ profiles obtained from

Equation [1] are shown in Figure 3c. Note that these represent the overall changes in the cell's enthalpy (without any distinction of the underlying steps). Since the $U_{H}$ profile on discharge is well separated from the one on charge, as indicated by a double-headed arrow in Figure 3c, the condition in Equation [4] cannot be fulfilled over the first cycle. Hence, there is a net change in enthalpy, as further schematized in Supp. Figure 1b. In other words, the material does not return to the pristine state after a full charge-discharge cycle, consistent with previous characterizations..$^{23,25,32}$ In summary, the large heat release during the voltage plateau may be due to the underlying rearrangement step that accompanies anionic oxidation. The subsequent discharge follows a thermodynamically different path.

[Figure 3]

Figure 3 | Isothermal calorimetry characterization of the full first cycle. (a) Voltage profile (blue) and (b) heat flow profile (orange) for the staircase-like first charge, followed by an S-shaped sloped discharge. An unintended interrupt of 104.4 mins (marked by *) occurred during the anionic oxidation plateau, which is further described in Supp. Figure 6. The steep rise in heat release at the end of the plateau is unrelated to the current interrupt and may be explained by the expected rise in 
irreversible heat contribution due to poor kinetics when the reaction approaches its end. Other possibilities include partial oxygen release ${ }^{25}$ and/or side reactions, possibly correlated with the small voltage feature near 4.5 V. The cell used here was the same as the one in Figure 2 on partial cycling (cationic redox only), which is overlaid here for comparison with the full cycle. (c) The calculated enthalpy potential profiles (pink) are shown along with the cell voltage profile over the complete first cycle. A double-headed green arrow highlights the separation between charge versus discharge enthalpy potentials.

\section{Isothermal calorimetry results for 'activated' LRSO}

After some formation cycles, LRSO's sloped S-shaped voltage profiles stabilize, with nearly equal capacity contributions from cationic and anionic redox processes that take place at low and high potentials respectively., ${ }^{9,23}$ To understand the electrochemical mechanism of this 'activated' phase, isothermal calorimetry was performed at different rates ranging from $\mathrm{C} / 50$ to $\mathrm{C} / 5$. The resulting voltage profiles exhibit a significant voltage hysteresis that does not diminish by lowering the current (Figure 4a). The heat flow for the slowest rate of $\mathrm{C} / 50$ (Figure 4b) is not symmetric and remains largely above zero for the entire cycle, unlike the classical cationic redox in previous Figure 2.

Again, the $U_{H}$ profiles were calculated from

Equation [1], as shown in Figure 4b for C/50 (other rates in Supp. Figure 8, comparison with initial cycle in Supp. Figure 9). Interestingly, the $U_{H}$ profiles on charge vs. discharge are much closer in comparison to the hysteretic voltage profiles. A larger heat generation on discharge indicates that the reaction's enthalpy potential is located further away from the discharge voltage than the charge voltage. Note that, despite a low rate of $\mathrm{C} / 50$, the $U_{H}$ profiles on charge and discharge do not fully superimpose, especially in the second half of SoCs (anionic redox region), thus reflecting a slight path dependence in the cell's enthalpy, as also schematized in Supp. Figure 1c. This effect was consistently observed for several cells (Supp. Figure 10). Whether this slight mismatch is due to kinetic or thermodynamic reasons can be understood from Figure 4c. On either 
charge or discharge, the $U_{H}$ profiles obtained at different rates converge over almost the entire SoC range, except possible heat of mixing effects at the end of charge and the beginning of discharge (highlighted with green backgrounds). Therefore, enthalpy-wise, the charge and discharge paths are rate-independent over the range of rates studied herein (from $\mathrm{C} / 50$ to $\mathrm{C} / 5$ ). Moreover, these paths are slightly dissimilar as they do not converge to a single unique curve. 'Activated' LRSO thus follows metastable paths having slightly different enthalpies on charge vs. discharge for the same SoC. Therefore, the overall Li content is no longer the unique reaction coordinate to fully describe the system, as discussed later.

Nevertheless, since 'activated' LRSO shows fairly stable heat profiles with negligible voltage fade in consecutive cycles (Supp. Figure 11), the $U_{H}$ profiles should satisfy Equation [4], despite being slightly different on charge vs. discharge, as also schematized in Supp. Figure 1c. This can be verified from Figure 4d showing the match between net heat and work (over complete cycles). The extra work needed due to voltage hysteresis is therefore dissipated as heat. The zerocurrent intercept in Figure $4 \mathrm{~d}$ is large $\left(\sim 70.4 \mathrm{Wh} \mathrm{kg}^{-1}\right)$, unlike Figure 2d, because of the large quasi-static hysteresis. It is key here to note that this significant dissipation can be viewed, according to Equation [7], as entropy production and implies that the underlying steps are far away from equilibrium even at the lowest rates (further discussion with Supp. Figure 2). Entropy production (the value of the integrals in Figure 4d) should ideally vanish to zero at infinitesimally small rates at which all the underlying steps would be near equilibrium. However, demonstrating this is not trivial simply due to prohibitively long experiments as well as the interfering sidereactions at extremely low C-rates. Such non-equilibrium arguments also explain the continuous voltage relaxation observed for LRSO under open circuit conditions, despite long time periods (24 hours $)^{9}$, because some of the underlying steps have slow equilibration dynamics with very large time constants. Since similar slow dynamics plague Li-rich $\mathrm{NMC}^{10}$, equilibrium is never achieved and the reported values of the entropic term $\frac{\partial U}{\partial T}$ are invalid as they violate Equation [6] during the 
sloped cycles $^{31}$. Likewise, distinguishing the true equilibrium potential $U$ (unique) from pathdependent metastable equilibrium potentials (non-unique) requires further thermodynamics development.

Overall, it is clear that voltage hysteresis is linked to non-equilibrium entropy production, which can be quantified over a complete cycle as the net dissipated heat or lost electrical work. However, further deconvolution of the heat flow rate into entropy change and entropy production rates, as per Equation [5], is not possible without precise knowledge of the underlying mechanism. Therefore, a multi-step mechanism consistent with calorimetry results is presented later.

[Figure 4]

Figure 4 | Isothermal calorimetry characterization of 'activated' LRSO. The hysteretic voltage profiles (a) obtained with LRSO cycled inside the calorimeter using CCCV protocols. $\boldsymbol{d} \boldsymbol{Q} / \boldsymbol{d} \boldsymbol{V}$ curves are shown in Supp. Figure 7. The experiments were performed after a few formation cycles in the full potential range leading to complete 'activation'. The quasi-static hysteresis appears to be larger in the higher potential anionic redox range. For $\mathrm{C} / 50$ (b), the measured profiles of cell voltage (blue) and heat flow rate (orange) that were used to obtain the $\boldsymbol{U}_{\boldsymbol{H}}$ profiles (pink). A zoom in inset shows the slight differences in $\boldsymbol{U}_{\boldsymbol{H}}$ profiles over the high potential region. (c) Convergence of $\boldsymbol{U}_{\boldsymbol{H}}$ profiles obtained at different rates for charge (top) and on discharge (bottom), with green backgrounds highlighting the deviations possibly due to heat of mixing. (d) As a function of C-rate, the comparison of lost electrical work with dissipated waste heat over a complete charge-discharge cycle obtained by integrating respectively the cell voltage and the heat flow rate. These integrals scale linearly with current, with the slope and intercept respectively related to the "SoC averaged" values of $\boldsymbol{R}_{\text {int }}$ and $\boldsymbol{V}_{\text {hyst }}$ in Equation [10]Error! Reference source not found.

Voltage window opening experiments were next performed. Figure 5a reveals that charging to high potentials, particularly beyond $50 \% \mathrm{SoC}$, gradually triggers voltage hysteresis. Akin to the discharge voltage profiles that are path dependent (i.e. they depend on the extent of charging), the discharge heat flow profiles (Figure 5b) also exhibit path dependence. Consistent with voltage hysteresis, discharging from the fully charged state results in a larger heat generation over a broad range of SoCs (denoted by arrows) compared to discharge from partially charged states. The 
corresponding $U_{H}$ discharge profiles (Figure $5 c$ ) in the lower half of SoCs (cationic redox ${ }^{23}$ ) converge independently of the charging history. In contrast, for the upper half of SoCs (anionic redox $^{23}$ ), the discharge $U_{H}$ curves gradually evolve as a function of the extent of charge, thus highlighting the distinctive role of the anionic redox process in causing voltage hysteresis. Lastly, the net heat and work over complete cycles are compared in Figure $5 \mathrm{~d}$ as a function of the charging SoC. They again match with each other, consistent with Equation [7]. Instead of a linear trend with current that is expected for path reversible intercalation materials, a non-linear rise is observed in Figure $5 \mathrm{~d}$ because of gradually worsening voltage hysteresis as the charging voltage window is opened. In light of these unusual observations triggered by high potential charging, a multi-step mechanism for the anionic redox process is proposed next.

[Figure 5]

Figure 5 | Isothermal calorimetry for charge voltage window opening of 'activated' LRSO. (a) Voltages profiles (blue). $\boldsymbol{d Q} / \boldsymbol{d V}$ curves are shown in Supp. Figure 12. (b) Heat flow profiles (orange) on discharge only. Complete heat profiles in Supp. Figure 13. (c) The calculated $\boldsymbol{U}_{\boldsymbol{H}}$ profiles (pink, for discharge only). $\boldsymbol{U}_{\boldsymbol{H}}$ profiles on charge are identical as expected (see Supp. Figure 14). A dashed curve in the background shows the full range charge-discharge voltage profile. The initial kinks in $\boldsymbol{U}_{\boldsymbol{H}}$ profiles at the onset of discharges is likely due to heat of mixing, as also observed in Figure 4c. (d) As a function of SoC to which the cell is charged, a comparison of lost electrical work with dissipated waste heat over a complete charge-discharge cycle obtained by integrating respectively the cell voltage and the heat flow rate. This non-linear trend was verified with a second cell (Supp. Figure 15).

\section{Proposed multi-step mechanism for hysteretic anionic redox}

The mechanism behind the hysteretic anionic redox process in 'activated' LRSO can now qualitatively be understood in light of the above calorimetry results. From the point of view of local structure, cationic redox can be treated as a single electrochemical step, therefore free from quasistatic voltage hysteresis (Figure 6a). On the other hand, anionic redox can be described as a multi- 
step process, consisting of sequential redox (electrochemical) and rearrangement (chemical) steps,

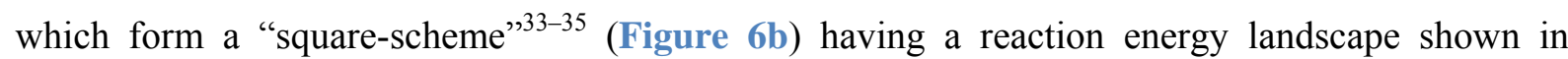
Figure 6c. On charge, the electrochemical oxidation of anions is followed by a stabilizing structural rearrangement that could either enlist the distortion of octahedral symmetry leading to $\mathrm{O}-\mathrm{O}$ shortening $^{7,23}$, metal-ion migration ${ }^{6,8,11,20,21}$, or a layer stacking change ${ }^{14,15}$. Owing to this structural modification, a different redox step is needed on discharge, which is again followed by a stabilizing rearrangement to the original state with reduced anions. Interestingly, these steps are analogous to the "square-scheme" mechanism that causes voltage hysteresis in certain molecular machines. ${ }^{34-36}$ Note that the four elementary steps are thermodynamically constrained as they form a closed cycle, but they do not necessarily take place close to equilibrium. A complementary experiment of gradually opening the discharge voltage window (Supp. Figure 16) fully supports the proposed mechanism.

Such a multi-step mechanism allows for non-equilibrium to exist if the kinetics of some of the elementary steps, most likely the chemical rearrangements steps, is slow enough (much slower than the C-rates used). In such a non-equilibrium case, the overall Li content of the electrode material is not the only reaction coordinate (the other coordinates being related to species repartitioning). The persistence of metastable paths can thus be explained, such that, independent of current, charge predominantly occurs via the upper path in Figure 6b and discharge via the lower one. Therefore, the non-equilibrium cell potentials during charge and discharge are respectively closer to the thermodynamic potentials $U_{\mathrm{ch}}^{\mathrm{A}}$ and $U_{\mathrm{dis}}^{\mathrm{A}}$ of the two redox steps, hence giving rise to voltage hysteresis. This hysteresis leads, by definition, to dissipative entropy production ${ }^{29}$, whose value on charge and discharge is in part related to the Gibbs free energy of reaction of the respective rearrangement step (Figure 6c). Nevertheless, the exact mathematical form of entropy production, as described along-with Supp. Figure 2, depends on the underlying thermodynamics and kinetics of the elementary steps that are, unfortunately, not easy to parametrize for LRSO. Thus, novel 
methods need to be developed for determining the entropy change for such path-dependent materials that show continuous OCV drift. Note that true equilibrium theoretically does exist in the scheme of Figure $6 \mathrm{~b}$, i.e. when the four steps occur in unison, but this situation is never achieved at practical C-rates, hence leading to metastability. This is the key difference in comparison to cationic redox, which also involves local structural changes $\left(\mathrm{RuO}_{6}\right.$ octahedral expansion/contraction and $\mathrm{Ru}-\mathrm{Ru}$ distance change). However, these changes are energetically small and/or kinetically fast, and therefore always in equilibrium with the redox step.

Concerning practical applications, the obstacle of quasi-static voltage hysteresis in Li-rich electrodes can only be overcome by addressing directly the anionic redox process that triggers atomic motion that is either kinetically slow and/or thermodynamically large in energy penalty, hence causing metastable reaction pathways and voltage hysteresis. Towards these goals, revisiting anionic redox chemistry in chalcogenides ${ }^{37}$, owing to their lower chemical hardness, may prove beneficial for fundamental understanding of the root cause behind hysteresis. Likewise, hysteresisfree anionic redox in $\mathrm{Na}_{4 / 7}\left[\square_{1 / 7} \mathrm{Mn}_{6 / 7}\right] \mathrm{O}_{2}$ calls for further investigation of Na-based cathodes. ${ }^{38}$

\section{[Figure 6]}

Figure 6 | Proposed mechanism for hysteretic bulk anionic redox in 'activated' LRSO. (a) Cationic redox can be described as a single step electrochemical reaction. $\mathbf{C}$ and $\mathbf{C}^{+}$are the reduced and oxidized cationic species (e.g. $\mathrm{Ru}^{4+}$ and $\mathrm{Ru}^{5+}$ ). (b) Anionic redox is described by a multi-step "square-scheme" mechanism. A denotes the anionic species in reduced state (e.g. $\mathrm{O}^{2-}$ ) that upon oxidation forms unstable $\mathrm{A}^{+}$(e.g. $\mathrm{O}^{n-}$ with $n<2$ ). Structural reorganization spontaneously takes place to form stabilized $\mathrm{A}_{r}{ }^{+}$(e.g. dimerized $\mathrm{O}-\mathrm{O}^{2 n-}$ with possible metal-ion migration ${ }^{6,7}$ ). Reduction

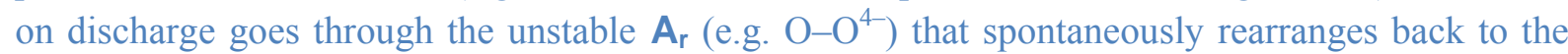
original state. $\mathbf{A}^{+}$and $\mathbf{A}_{\mathrm{r}}$ are therefore the high-energy intermediates for the two respective paths. Note that electrons and $\mathrm{Li}$ ions are not explicitly shown in these schemes. Also, the cationic and anionic reactions may occur in a partially overlapped fashion depending of the precise kinetic and thermodynamic parameters. (c) A schematic reaction energy landscape, highlighting (i) the downhill nature of chemical (C) rearrangement steps, and (ii) their associated kinetic barriers. These two conditions explain path-dependence. If the larger heat generation experimentally observed on discharge is assumed primarily due to entropy production (i.e. minor contributions from reversible entropy), then $\Delta \boldsymbol{G}_{\mathbf{d i s}}^{\mathbf{r}}$ in 'activated' LRSO is expected to be larger than $\Delta \boldsymbol{G}_{\mathbf{c h}}^{\mathbf{r}}$, implying that the O-O 


\section{dissociation is energy-wise costlier than its formation.}

The above mechanism bears a similarity with the previously proposed "activated bonding change" model for hysteretic hydrogen-containing carbons ${ }^{39,40}$, or the phase-change-based model for hysteretic Li-rich $\mathrm{NMC}^{10,24}$, or the model based on limited ionic mobility for certain hysteretic displacement reactions ${ }^{41}$. All these models lead to a non-equilibrium situation under operating conditions, thus making it necessary to consider the heat associated with bonding change or phase change, like previously done for hysteretic carbons. ${ }^{42}$

Lastly, apart from providing mechanistic insights about anionic redox in Li-rich electrodes, this work provides a new direction in the characterization of batteries with isothermal calorimetry. A particular highlight is the importance of $U_{H}$, the enthalpy potential, that can greatly help in predicting the total heat generation needed for battery thermal management. Therefore, we recommend the measurement of $U_{H}$, especially for battery materials that either show large entropy changes or a quasi-static voltage hysteresis (silicon, graphite, $\mathrm{LiFePO}_{4}, \mathrm{Li}-\mathrm{S}, \mathrm{Li}-\mathrm{O}_{2}$, and conversion electrodes). The non-equilibrium thermodynamics approach developed herein could be extended to electrochemical systems beyond batteries, such as water-splitting catalysts showing large overpotentials or to hysteretic solar cells.

\section{Conclusion}

In summary, isothermal calorimetry has elucidated the general thermodynamic mechanism governing the 'model' Li-rich $\mathrm{Li}_{2} \mathrm{Ru}_{0.75} \mathrm{Sn}_{0.25} \mathrm{O}_{3}$ (LRSO) cathode's electrochemistry. We proved that charging LRSO through the anionic oxidation plateau leads to heat generation and a permanent change of the material, such that it displays sloped and hysteretic voltage profiles with reversible oxygen redox chemistry in the subsequent cycles. In a thermodynamically consistent manner, we demonstrated that the electrical work lost due to such voltage hysteresis is dissipated as waste heat. It is now clear that (i) metastable paths persist even under quasi-static conditions and that (ii) 
voltage hysteresis is directly related to non-equilibrium entropy production instead of simply originating from large differences in the enthalpy of charge vs. discharge paths. Far-fromequilibrium is therefore an essential condition for quasi-static voltage hysteresis, which renders the estimation of true equilibrium properties quite difficult. The extension of this work to the 'practical' Li-rich NMC, among other anionic redox cathodes, is imminent and similar results are expected. Furthermore, the implementation of model-based simulations could help in generalizing the multistep "square-scheme" model proposed herein. Such fundamental understanding is necessary for designing effective mitigation approaches that can lead to the eventual elimination of voltage hysteresis. In the broader context, this new direction for battery characterization using isothermal calorimetry would encourage the electrochemistry community to further exploit this tool as well as to adopt the concepts of enthalpy potential and non-equilibrium entropy production. From an applied perspective, the methodology developed herein calls for revisiting some known hysteretic materials (silicon, conversion-based) and would also help in screening emerging materials for minimizing heat generation. 


\section{Methods}

Energy balance for an electrochemical cell. The general energy balance for an electrochemical system was derived by Newman and co-workers. ${ }^{27,43,44}$ For a thermally thin electrochemical cell in isothermal (constant temperature) and isobaric (constant pressure) conditions, the heat generation rate $\left.\dot{q}\right|_{T, P}$ (if heat of mixing is neglected ${ }^{27,28}$ ) is given by

$$
\left.\dot{q}\right|_{T, P}=I(V-U)+I T \frac{\partial U}{\partial T}+\sum_{i} \Delta H_{i} r_{i}
$$

Equation [8]

where $\left.\dot{q}\right|_{T, P}$ is positive if heat is released by the cell, $I$ is the current (positive for charging), $V$ is the cell voltage, $U$ is the equilibrium potential, $T$ is the temperature, $P$ is the surrounding pressure, and $\Delta H_{i}$ is the enthalpy of reaction of a chemical reaction $i$ (other than the main electrochemical reaction) occurring at a rate $r_{i}$. Recognizing the first term as irreversible heat generation due to overpotential, the second term as reversible entropic heat, and the last term as parasitic heat due to side reactions, Equation [8]Error! Reference source not found. can also be equivalently formulated $\mathrm{as}^{26}$

$$
\left.\dot{q}\right|_{T, P}=I \eta+\frac{I T}{n F}\left(\frac{d S_{+}}{d x}-\tilde{s}_{L i}\right)+\dot{q}_{p}
$$

where $\eta$ is the cell's overpotential (the product $I \eta$ is always positive), $n$ is the number of electrons involved in electrode reaction, $F$ is the Faraday's constant, $x$ is the stoichiometric content of $\mathrm{Li}$ in the positive electrode material, $S_{+}$is the total entropy of the positive electrode material at a given $x, \tilde{s}_{L i}$ is the molar entropy of Li metal negative electrode, and $\dot{q}_{p}$ is the parasitic heat flow due 
to other chemical reactions. These formulations have extensively been used for investigating the entropic heat of intercalation materials ${ }^{45}$ and more recently the parasitic heat in Li-ion cells ${ }^{30,46-49}$. For quantifying the entropy change, one possibility (potentiometric method) is to perform temperature-dependent equilibrium potential measurements for directly determining $\frac{\partial U}{\partial T}$ in Equation [8]Error! Reference source not found.. ${ }^{28,50-53}$ Another option (calorimetric method) is to measure $V$, $I$, and $\left.\dot{q}\right|_{T, P}$ over a charge-discharge cycle and obtain the terms in Equation [9] while considering these terms to be path independent (same on charge vs. discharge), which is a reasonable assumption for classical intercalation materials only. ${ }^{26,30,49}$ Lastly, a method called electrothermal impedance spectroscopy (ETIS) has also recently been developed. ${ }^{54}$ To understand the effect of quasi-static voltage hysteresis, the irreversible heat term can be empirically modelled, when $I$ is relatively low, as ${ }^{49}$

$$
I \eta=I^{2} R_{\text {int }}+|I| V_{\text {hyst }}
$$

where $R_{\text {int }}$ is the cell's internal resistance and $V_{\text {hyst }}$ the inherent quasi-static (extrapolated to zero-current) voltage hysteresis. This equation was successfully applied to empirically model the heat flow in $\mathrm{LiNi}_{0.4} \mathrm{Mn}_{0.4} \mathrm{Co}_{0.2} \mathrm{O}_{2}$ /graphite cells by including a small $( \pm \sim 4 \mathrm{mV})$ voltage hysteresis equally on charge and discharge and by considering $R_{\text {int }}$ as a function of SoC only. ${ }^{49}$ However for the Li-rich electrodes, this approach is not suitable because (i) they show a much larger hysteresis (both Li-rich NMC and LRSO that are of interest in this work) and (ii) their $R_{\text {int }}$ is known to vary differently on charge vs. discharge with SoC. ${ }^{5,9}$ Likewise, $V_{\text {hyst }}$ can be expected to differ as a function of cell potential on charge vs. discharge in these highly path-dependent materials. In other words, the position of $U$ in hysteretic Li-rich electrodes cannot simply be assumed in the middle of the charge and discharge profiles, unlike in classical intercalation materials. We therefore use a 
different approach herein that is based on "enthalpy potential", as described in the main text.

Material synthesis and electrode fabrication. Single-phase $\mathrm{Li}_{2} \mathrm{Ru}_{0.75} \mathrm{Sn}_{0.25} \mathrm{O}_{3}$ (LRSO) samples, as determined by X-ray powder diffraction, were obtained by solid-state synthesis reported elsewhere. ${ }^{25}$ The Bellcore plastic method $^{55}$ was used to prepare high-porosity self-standing electrodes, as described in detail previously. ${ }^{9}$ The final electrode film was composed of $73 \%$ (by weight) active material, 9\% carbon SP (MM, Belgium), and 18\% binder - poly(vinylidene fluorideco-hexafluoropropylene) (PVDF-HFP) (Arkema). The film was designed for an active material loading of $\sim 20 \mathrm{mg} \mathrm{cm}^{-2}, \sim 200 \mu \mathrm{m}$ film-thickness, and $\sim 60 \%$ porosity. Such a relatively high loading was needed to generate reliable amounts of heat flow from the coin cells. Owing to the high porosity of carefully designed electrodes, in addition to the relatively low C-rates studied herein, the liquid-phase transport limitations (and their associated entropy production ${ }^{56,57}$ ) are expected to remain very low compared to those in the solid phase, as also suggested via previous modelling ${ }^{57,58}$ investigations.

Coin-cell assembly. Using $\sim 1 \mathrm{~cm}^{2}$ circular discs of this electrode film, 2032 coin-type cells with Li metal foil as the negative electrode were assembled in an argon-filled glove-box with two layers of $12 \mu \mathrm{m}$ polyethylene film coated with $4 \mu \mathrm{m}$ alumina (W-SCOPE separators) along with one layer of a Whatman ${ }^{\mathrm{TM}} \mathrm{GF} / \mathrm{D}$ borosilicate glass microfiber sheet (GE Healthcare Life Sciences). The electrolyte used was LP100 (Merck) having $1 \mathrm{M} \mathrm{LiPF}_{6}$ in ethylene carbonate : propylene carbonate : dimethyl carbonate in a $1: 1: 3$ volume ratio. No stress build-up (mechanical work) is expected to occur inside the cell owing to LRSO's very small lattice volume change (maximum $3.7 \%{ }^{25}$, along with the use of a soft and deformable glass microfiber separator that can accommodate any residual strain. The $\mathrm{C}$-rate in this work is defined such that $1 \mathrm{C}$ corresponds to the removal of $1 \mathrm{Li}$ per formula unit of LRSO in one hour $\left(1 \mathrm{C}=160.13 \mathrm{~mA} \mathrm{~g}^{-1}\right)$. 
Isothermal Calorimetry. Depending on the experiment, the cells were pre-cycled at room temperature before being transferred into a TAM III Microcalorimeter (TA Instruments: stability \pm $0.0001{ }^{\circ} \mathrm{C}$, accuracy $\pm 1 \mu \mathrm{W}$, precision $\pm 10 \mathrm{nW}$ ) for isothermal calorimetry measurements at $40{ }^{\circ} \mathrm{C}$. A few formation cycles were again carried out once the cells were inside the calorimeter. Such precycling allows any possible side-reactions, such as gassing and/or surface stabilization in initial cycles, to reach to a nearly steady state. The baseline drift over the course of the experiments did not exceed $\pm 0.5 \mu \mathrm{W}$. The time constant of the calorimeter is about 5 minutes. All specifications and information regarding microcalorimetry calibration, cell connections, and operation procedures can be found in previous literature..$^{30,46-49}$ The electrochemical cycling was performed with a Maccor 4000 series cycler connected to the cells inside the calorimeter. Depending on the experiment, either a constant current (CC) or a constant current constant voltage (CCCV) protocol was used. The voltage hold of the CCCV step was terminated when current decayed to a low value of $\mathrm{C} / 100$. The observed heat flow, defined positive when heat is generated by the cell, mainly arises from the positive electrode. A constant and small value of background heat of $+3.4 \mu \mathrm{W}$, accounting for parasitic reactions, was subtracted from the raw measured heat flow values, irrespective of cationic or anionic redox regime. Such an assumption of constant parasitic heat is acceptable given the scope of this study. Still, it must be kept in mind that parasitic heat depends on the complex relationship among the cathode, the anode, the electrolyte, the operating conditions (cell voltage etc.), the cell history over the course of the experiment, besides the continuous corrosion of inactive cell parts. 


\section{Data availability.}

\section{* Corresponding authors:}

C. Delacourt: charles.delacourt@u-picardie.fr

J.-M. Tarascon: jean-marie.tarascon@college-de-france.fr

\section{Acknowledgements}

\section{Author Contributions} equipment used in this work.

J.M.T. and G.A. acknowledge the funding from the European Research Council (ERC) (FP/2014)/ERC Grant-Project 670116-ARPEMA. S.L.G thanks NSERC and the Walter C. Sumner Foundation for funding. We are grateful to Dr. Jeff Dahn for providing access to some of the

G.A. and S.L.G. conceived the idea. G.A. prepared the electrochemical cells and S.L.G. performed the isothermal calorimetry experiments. G.A. and C.D. performed the thermodynamic analysis. J.M.T. supervised the project. G.A. wrote the paper, with contributions from all authors.

\section{Competing Financial Interests}

The authors declare no competing financial interests. 


\section{References}

1. Zheng, J. et al. Li- and Mn-Rich Cathode Materials: Challenges to Commercialization. Adv. Energy Mater. 1601284 (2016). doi:10.1002/aenm.201601284

2. Manthiram, A., Knight, J. C., Myung, S.-T., Oh, S.-M. \& Sun, Y.-K. Nickel-Rich and LithiumRich Layered Oxide Cathodes: Progress and Perspectives. Adv. Energy Mater. 6, 1501010 (2016).

3. Myung, S.-T. et al. Nickel-rich Layered Cathode Materials for Automotive Lithium-ion Batteries: Achievements and Perspectives. ACS Energy Lett. (2016). doi:10.1021/acsenergylett.6b00594

4. Luo, K. et al. Charge-compensation in 3d-transition-metal-oxide intercalation cathodes through the generation of localized electron holes on oxygen. Nat. Chem. 8, 684-691 (2016).

5. Assat, G. et al. Fundamental interplay between anionic/cationic redox governing the kinetics and thermodynamics of lithium-rich cathodes. Nat. Commun. 8, Article number: 2219 (2017).

6. Gent, W. E. et al. Coupling between oxygen redox and cation migration explains unusual electrochemistry in lithium-rich layered oxides. Nat. Commun. 8, (2017).

7. Assat, G. \& Tarascon, J.-M. Fundamental understanding and practical challenges of anionic redox activity in Li-ion batteries. Nat. Energy 1 (2018). doi:10.1038/s41560-018-0097-0

8. Croy, J. R. et al. Examining Hysteresis in Composite $x \mathrm{Li}_{2} \mathrm{MnO}_{3} \cdot(1-x) \mathrm{LiMO}_{2}$ Cathode Structures. J. Phys. Chem. C 117, 6525-6536 (2013).

9. Assat, G., Delacourt, C., Corte, D. A. D. \& Tarascon, J.-M. Editors' Choice-Practical Assessment of Anionic Redox in Li-Rich Layered Oxide Cathodes: A Mixed Blessing for High Energy Li-Ion Batteries. J. Electrochem. Soc. 163, A2965-A2976 (2016).

10. Dees, D. W. et al. Electrochemical Modeling and Performance of a Lithium- and ManganeseRich Layered Transition-Metal Oxide Positive Electrode. J. Electrochem. Soc. 162, A559A572 (2015).

11. Croy, J. R., Balasubramanian, M., Gallagher, K. G. \& Burrell, A. K. Review of the U.S. Department of Energy's 'Deep Dive' Effort to Understand Voltage Fade in Li- and Mn-Rich Cathodes. Acc. Chem. Res. 48, 2813-2821 (2015).

12. Meister, P. et al. Best Practice: Performance and Cost Evaluation of Lithium Ion Battery Active Materials with Special Emphasis on Energy Efficiency. Chem. Mater. 28, 7203-7217 (2016).

13. Ohzuku, T., Nagayama, M., Tsuji, K. \& Ariyoshi, K. High-capacity lithium insertion materials of lithium nickel manganese oxides for advanced lithium-ion batteries: toward rechargeable capacity more than $300 \mathrm{~mA} \mathrm{~h} \mathrm{~g}^{-1}$. J. Mater. Chem. 21, 10179 (2011). 
14. Maitra, U. et al. Oxygen redox chemistry without excess alkali-metal ions in $\mathrm{Na}_{2 / 3}\left[\mathrm{Mg}_{0.28} \mathrm{Mn}_{0.72}\right] \mathrm{O}_{2}$. Nat. Chem. (2018). doi:10.1038/nchem.2923

15. Mortemard de Boisse, B. et al. Intermediate honeycomb ordering to trigger oxygen redox chemistry in layered battery electrode. Nat. Commun. 7, 11397 (2016).

16. Yabuuchi, N. et al. Origin of stabilization and destabilization in solid-state redox reaction of oxide ions for lithium-ion batteries. Nat. Commun. 7, 13814 (2016).

17. A. House, R. et al. Lithium manganese oxyfluoride as a new cathode material exhibiting oxygen redox. Energy Environ. Sci. (2018). doi:10.1039/C7EE03195E

18. Croy, J. R., Gallagher, K. G., Balasubramanian, M., Long, B. R. \& Thackeray, M. M. Quantifying Hysteresis and Voltage Fade in $\mathrm{x} \mathrm{Li}_{2} \mathrm{MnO}_{3} \bullet(1-\mathrm{x}) \mathrm{LiMn}_{0.5} \mathrm{Ni}_{0.5} \mathrm{O}_{2}$ Electrodes as a Function of $\mathrm{Li}_{2} \mathrm{MnO}_{3}$ Content. J. Electrochem. Soc. 161, A318-A325 (2014).

19. Konishi, H. et al. Electrochemical reaction mechanisms under various charge-discharge operating conditions for $\mathrm{Li}_{1.2} \mathrm{Ni}_{0.13} \mathrm{Mn}_{0.54} \mathrm{Co}_{0.13} \mathrm{O}_{2}$ in a lithium-ion battery. J. Solid State Chem. 262, 294-300 (2018).

20. Dogan, F. et al. Re-entrant Lithium Local Environments and Defect Driven Electrochemistry of Li- and Mn-Rich Li-Ion Battery Cathodes. J. Am. Chem. Soc. 137, 2328-2335 (2015).

21. Kleiner, K. et al. Origin of High Capacity and Poor Cycling Stability of Li-Rich Layered Oxides: A Long-Duration in Situ Synchrotron Powder Diffraction Study. Chem. Mater. 30, 3656-3667 (2018).

22. Konishi, H. et al. Origin of hysteresis between charge and discharge processes in lithium-rich layer-structured cathode material for lithium-ion battery. J. Power Sources 298, 144-149 (2015).

23. Assat, G., Iadecola, A., Delacourt, C., Dedryvère, R. \& Tarascon, J.-M. Decoupling CationicAnionic Redox Processes in a Model Li-rich Cathode via Operando X-ray Absorption Spectroscopy. Chem. Mater. (2017). doi:10.1021/acs.chemmater.7b03434

24. Rinaldo, S. G. et al. Physical Theory of Voltage Fade in Lithium- and Manganese-Rich Transition Metal Oxides. J. Electrochem. Soc. 162, A897-A904 (2015).

25. Sathiya, M. et al. Reversible anionic redox chemistry in high-capacity layered-oxide electrodes. Nat. Mater. 12, 827-835 (2013).

26. Dahn, J. R. et al. Entropy of the intercalation compound $\mathrm{Li}_{\mathrm{x}} \mathrm{Mo}_{6} \mathrm{Se}_{8}$ from calorimetry of electrochemical cells. Phys. Rev. B 32, 3316-3318 (1985).

27. Thomas, K. E. \& Newman, J. Thermal Modeling of Porous Insertion Electrodes. J. Electrochem. Soc. 150, A176 (2003).

28. Thomas, K. E. \& Newman, J. Heats of mixing and of entropy in porous insertion electrodes. J. 
Power Sources 119-121, 844-849 (2003).

29. Kondepudi, D. \& Prigogine, I. Modern thermodynamics: from heat engines to dissipative structures. (Wiley \& Sons, 2015).

30. Glazier, S. L., Nelson, K. J., Allen, J. P., Li, J. \& Dahn, J. R. The Effect of Different Li(Ni $\mathrm{Ni}_{1-\mathrm{x}-}$ $\left.{ }_{\mathrm{y}} \mathrm{Mn}_{\mathrm{x}} \mathrm{Co}_{\mathrm{y}}\right) \mathrm{O}_{2}$ Positive Electrode Materials and Coatings on Parasitic Heat Flow as Measured by Isothermal Microcalorimetry, Ultra-High Precision Coulometry and Long Term Cycling. $J$. Electrochem. Soc. 164, A1203-A1212 (2017).

31. Shi, W. et al. The Effect of Entropy and Enthalpy Changes on the Thermal Behavior of Li-MnRich Layered Composite Cathode Materials. J. Electrochem. Soc. 163, A571-A577 (2016).

32. Salager, E. et al. Solid-State NMR of the Family of Positive Electrode Materials $\mathrm{Li}_{2} \mathrm{Ru}_{1-y} \mathrm{Sn}_{y} \mathrm{O}_{3}$ for Lithium-Ion Batteries. Chem. Mater. 26, 7009-7019 (2014).

33. Bard, A. J. \& Faulkner, L. R. Electrochemical methods: fundamentals and applications. (Wiley, 2001).

34. Sauvage, J.-P. Transition Metal-Containing Rotaxanes and Catenanes in Motion: Toward Molecular Machines and Motors. Acc. Chem. Res. 31, 611-619 (1998).

35. Sano, M. \& Taube, H. 'Molecular hysteresis' in an electrochemical system revisited. Inorg. Chem. 33, 705-709 (1994).

36. Sano, M. Mechanism of the molecular hysteresis. Polym. Adv. Technol. 6, 178-184 (1995).

37. Rouxel, J. Anion-Cation Redox Competition and the Formation of New Compounds in Highly Covalent Systems. Chem. - Eur. J. 2, 1053-1059 (1996).

38. Boisse, B. M. de et al. Highly Reversible Oxygen-Redox Chemistry at $4.1 \mathrm{~V}$ in $\mathrm{Na}_{4 / 7-x}\left[\square_{1 / 7} \mathrm{Mn}_{6 / 7}\right] \mathrm{O}_{2}$ (口: Mn Vacancy). Adv. Energy Mater. 0, 1800409

39. Zheng, T. \& Dahn, J. R. Hysteresis observed in quasi open-circuit voltage measurements of lithium insertion in hydrogen-containing carbons. J. Power Sources 68, 201-203 (1997).

40. Zheng, T., McKinnon, W. R. \& Dahn, J. R. Hysteresis during Lithium Insertion in HydrogenContaining Carbons. J. Electrochem. Soc. 143, 2137-2145 (1996).

41. Yu, H.-C. et al. Designing the next generation high capacity battery electrodes. Energy Environ. Sci. 7, 1760 (2014).

42. Inaba, M., Fujikawa, M., Abe, T. \& Ogumi, Z. Calorimetric Study on the Hysteresis in the Charge-Discharge Profiles of Mesocarbon Microbeads Heat-Treated at Low Temperatures. J. Electrochem. Soc. 147, 4008-4012 (2000).

43. Bernardi, D., Pawlikowski, E. \& Newman, J. A general energy balance for battery systems. J. Electrochem. Soc. 132, 5-12 (1985).

44. Rao, L. \& Newman, J. Heat-generation rate and general energy balance for insertion battery 
systems. J. Electrochem. Soc. 144, 2697-2704 (1997).

45. Bandhauer, T. M., Garimella, S. \& Fuller, T. F. A Critical Review of Thermal Issues in Lithium-Ion Batteries. J. Electrochem. Soc. 158, R1 (2011).

46. Krause, L. J., Jensen, L. D. \& Dahn, J. R. Measurement of Parasitic Reactions in Li Ion Cells by Electrochemical Calorimetry. J. Electrochem. Soc. 159, A937-A943 (2012).

47. Downie, L. E. \& Dahn, J. R. Determination of the Voltage Dependence of Parasitic Heat Flow in Lithium Ion Cells Using Isothermal Microcalorimetry. J. Electrochem. Soc. 161, A1782A1787 (2014).

48. Downie, L. E., Hyatt, S. R., Wright, A. T. B. \& Dahn, J. R. Determination of the Time Dependent Parasitic Heat Flow in Lithium Ion Cells Using Isothermal Microcalorimetry. $J$. Phys. Chem. C 118, 29533-29541 (2014).

49. Downie, L. E., Hyatt, S. R. \& Dahn, J. R. The Impact of Electrolyte Composition on Parasitic Reactions in Lithium Ion Cells Charged to 4.7 V Determined Using Isothermal Microcalorimetry. J. Electrochem. Soc. 163, A35-A42 (2016).

50. Wen, C. J. \& Huggins, R. A. Thermodynamic Study of the Lithium-Tin System. J. Electrochem. Soc. 128, 1181-1187 (1981).

51. Dahn, J. R. \& Haering, R. R. Entropy measurements on $\mathrm{Li}_{\mathrm{x}} \mathrm{TiS}_{2}$. Can. J. Phys. 61, 1093-1098 (1983).

52. Reynier, Y., Yazami, R. \& Fultz, B. The entropy and enthalpy of lithium intercalation into graphite. J. Power Sources 119-121, 850-855 (2003).

53. Forgez, C., Vinh Do, D., Friedrich, G., Morcrette, M. \& Delacourt, C. Thermal modeling of a cylindrical $\mathrm{LiFePO}_{4} /$ graphite lithium-ion battery. J. Power Sources 195, 2961-2968 (2010).

54. Schmidt, J. P., Weber, A. \& Ivers-Tiffée, E. A novel and precise measuring method for the entropy of lithium-ion cells: $\Delta \mathrm{S}$ via electrothermal impedance spectroscopy. Electrochimica Acta 137, 311-319 (2014).

55. Tarascon, J.-M., Gozdz, A. S., Schmutz, C., Shokoohi, F. \& Warren, P. C. Performance of Bellcore's plastic rechargeable Li-ion batteries. Solid State Ion. 86, 49-54 (1996).

56. Kjelstrup, S. \& Bedeaux, D. Non-equilibrium Thermodynamics of Heterogeneous Systems. (World Scientific, 2008).

57. Richter, F., Gunnarshaug, A., Burheim, O. S., Vie, P. J. S. \& Kjelstrup, S. Single Electrode Entropy Change for $\mathrm{LiCoO}_{2}$ Electrodes. ECS Trans. 80, 219-238 (2017).

58. Santhanagopalan, S., Guo, Q., Ramadass, P. \& White, R. E. Review of models for predicting the cycling performance of lithium ion batteries. J. Power Sources 156, 620-628 (2006).

59. Malifarge, S., Delobel, B. \& Delacourt, C. Experimental and Modeling Analysis of Graphite 
Electrodes with Various Thicknesses and Porosities for High-Energy-Density Li-Ion Batteries. J. Electrochem. Soc. 165, A1275-A1287 (2018). 
a

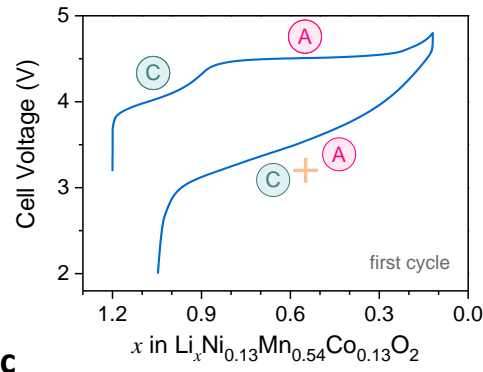

C

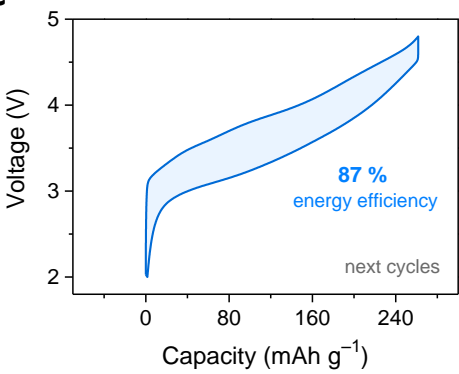

b
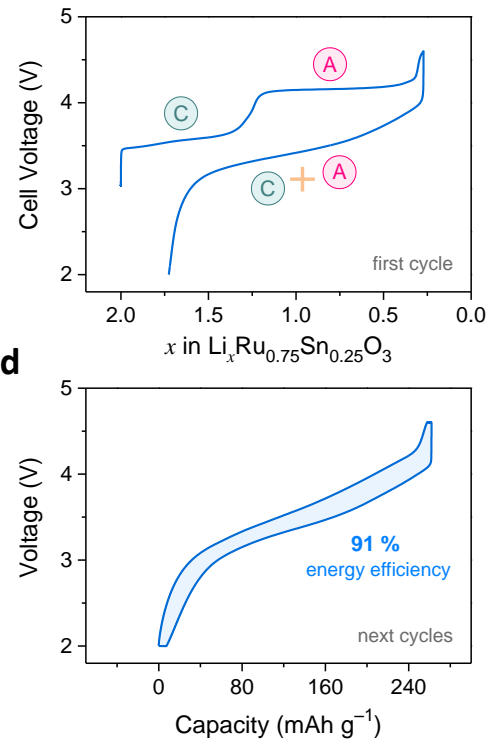
a
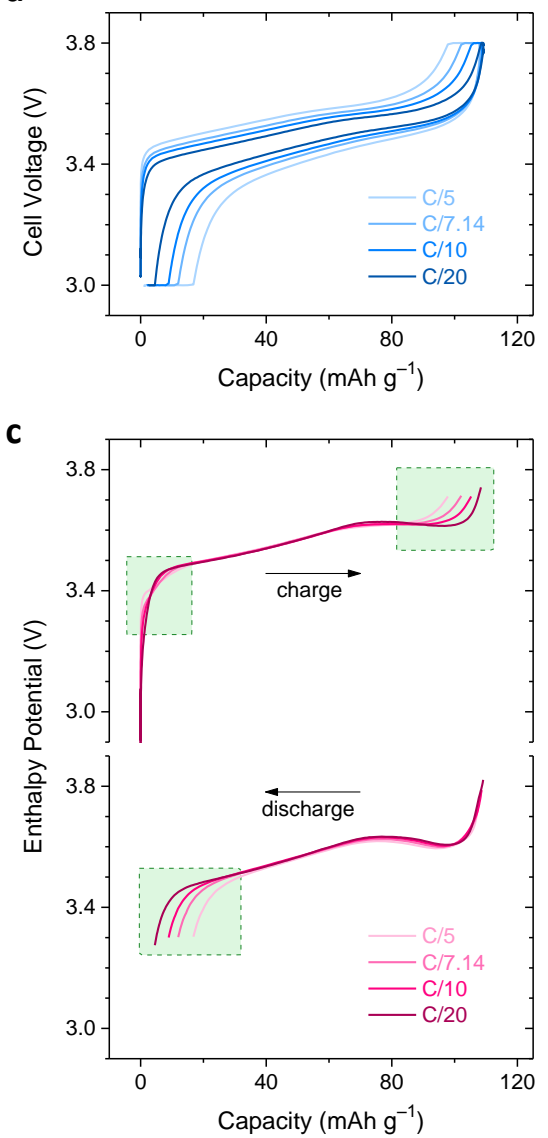

b
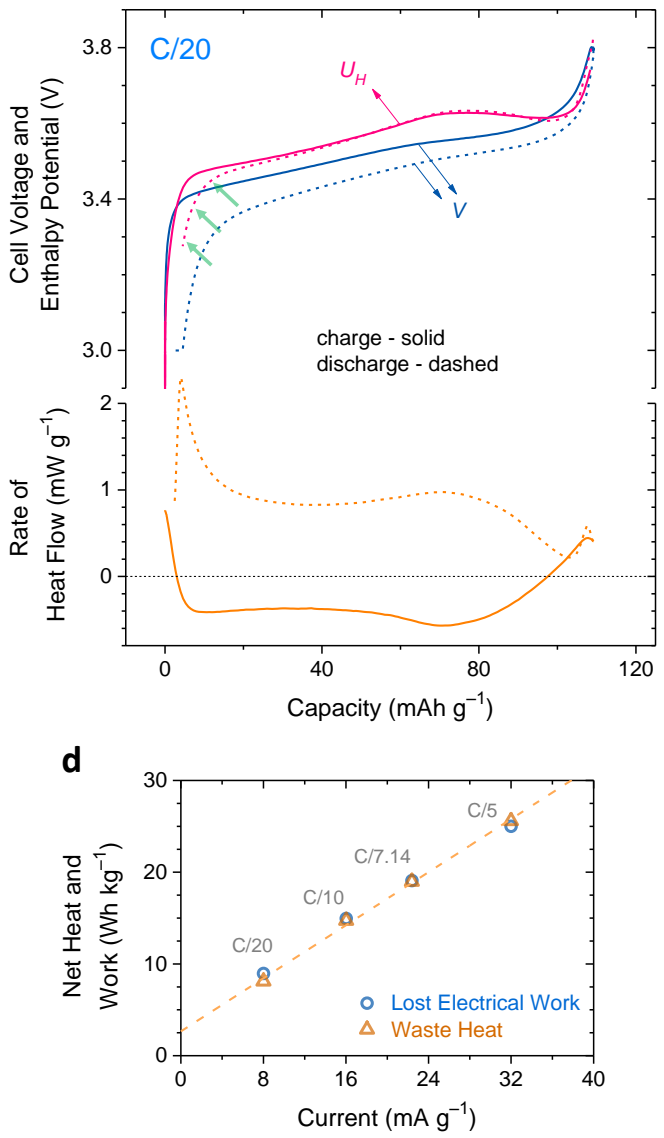


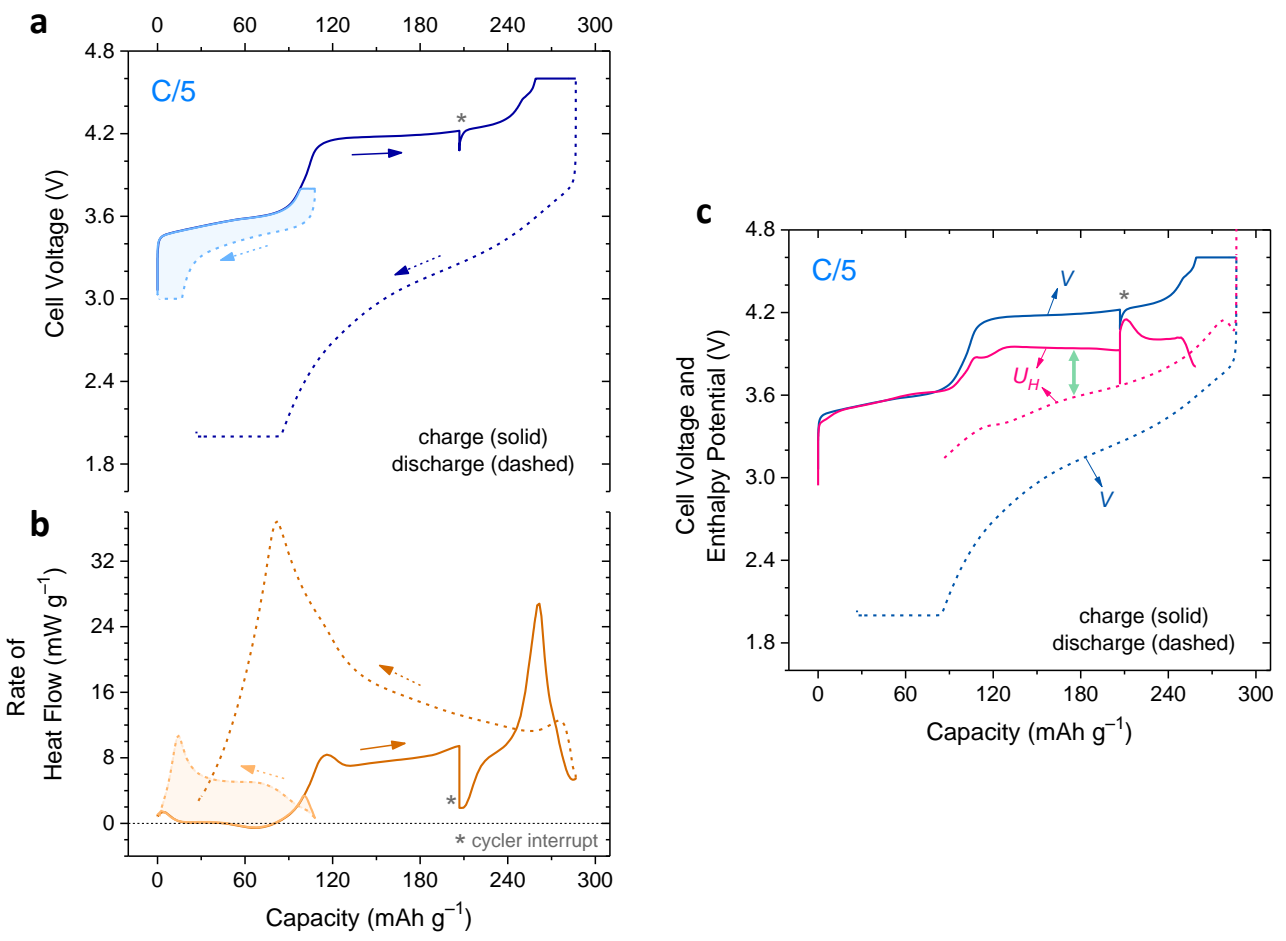


a

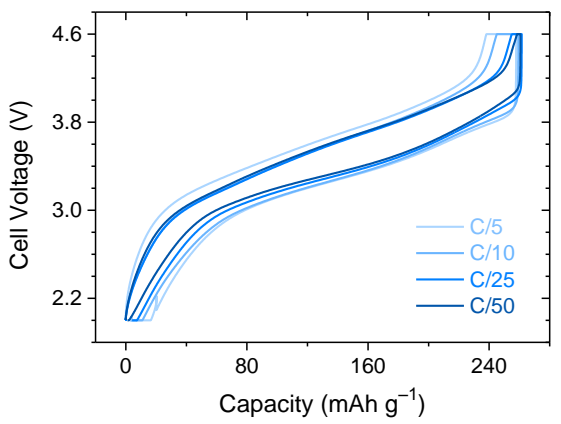

C

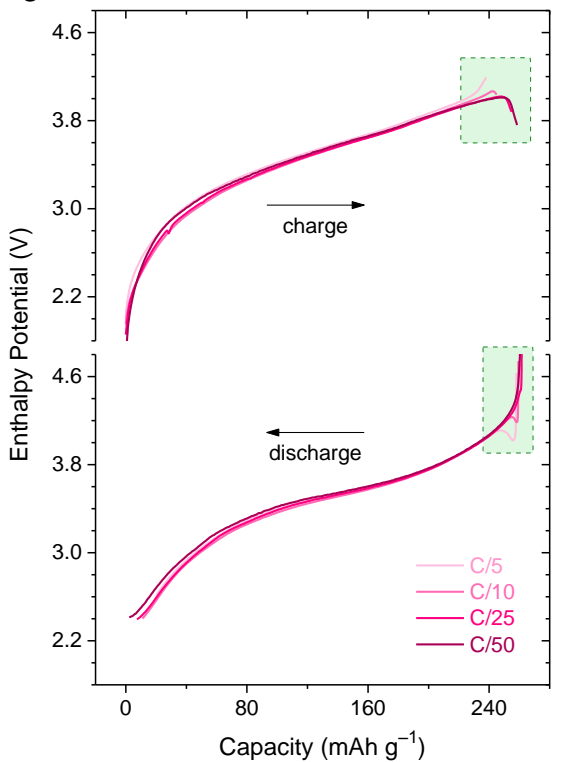

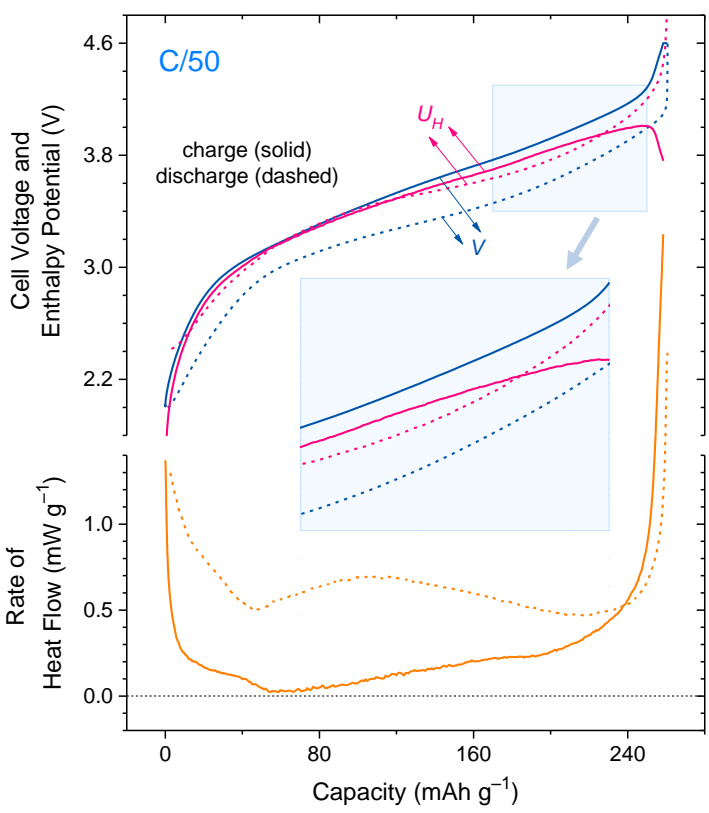

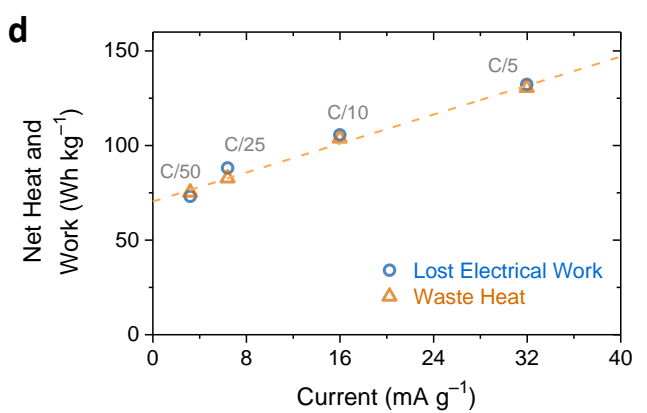



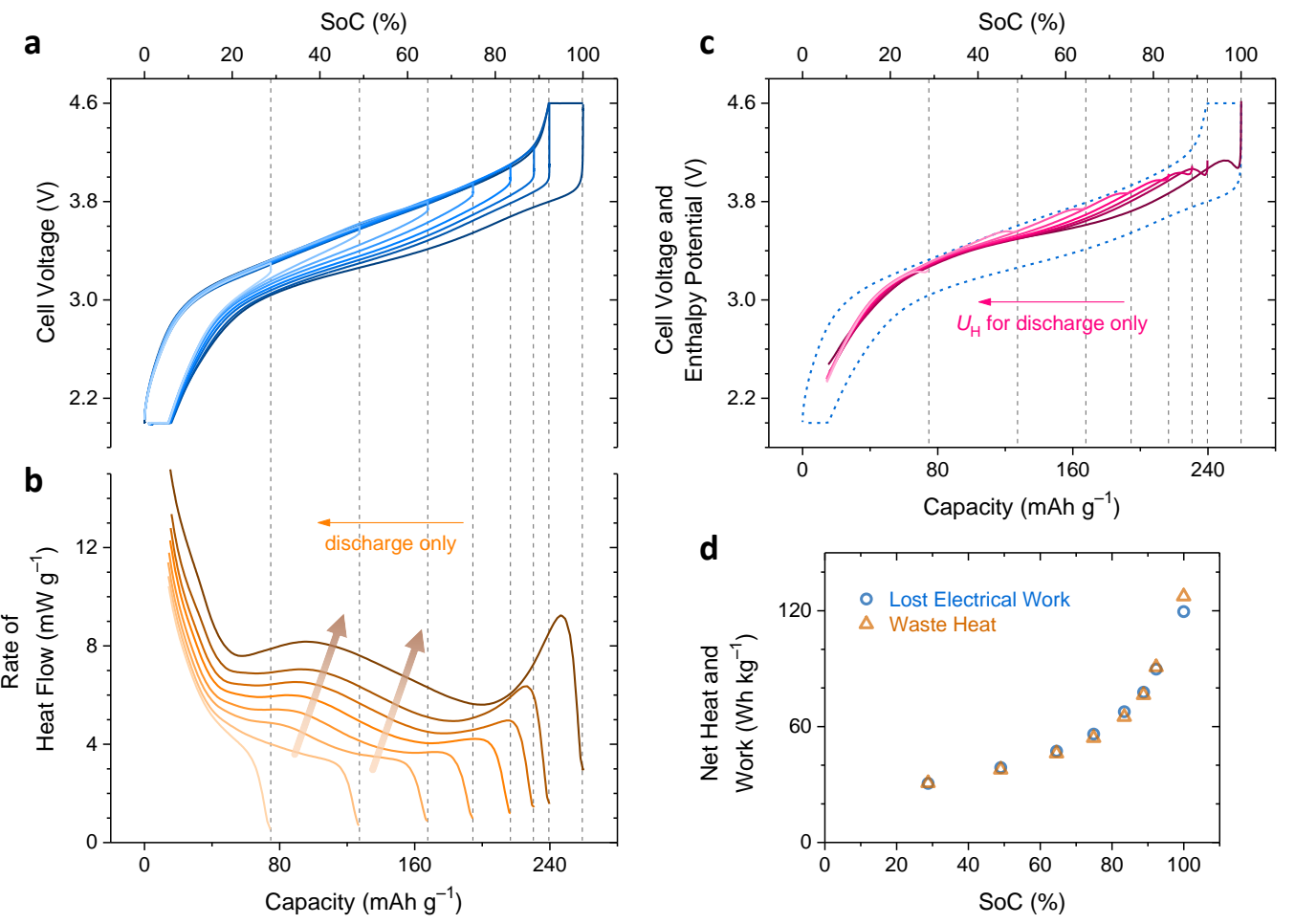


\section{a

$$
\mathrm{C} \underset{F U^{\mathrm{C}}}{\stackrel{\text { redox }}{\rightleftarrows}} \mathrm{C}^{+}
$$

\section{Cationic Redox}

b

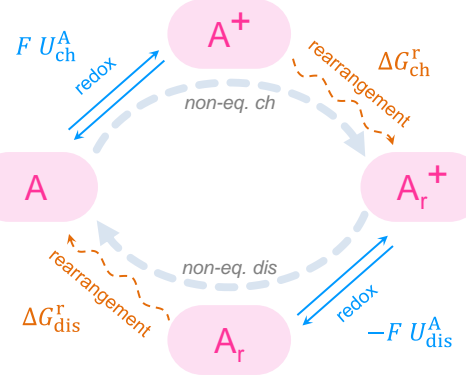

Anionic Redox
C

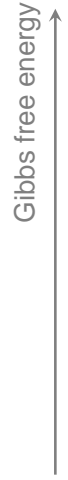

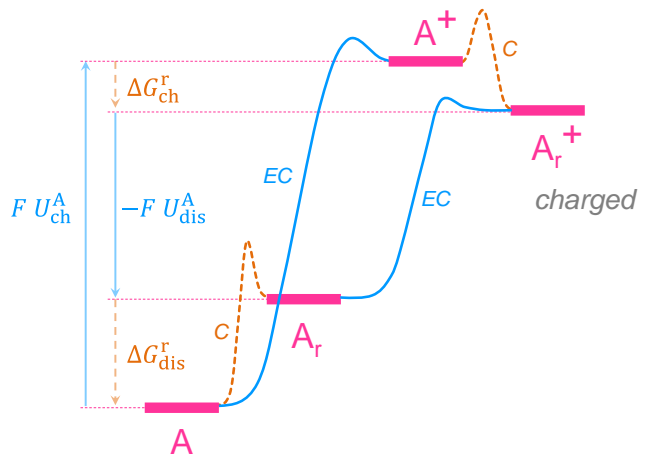

discharged 